\title{
Palliative Hypofractionated Radiotherapy in Advanced Squamous Cell Carcinoma of Head and Neck
}

\author{
Deepa Gautam ${ }^{1}$, Arati Shah ${ }^{2}$ \\ ${ }^{1}$ Department of Radiation Oncology, B.P. Koirala Memorial Cancer Hospital, Bharatpur, Nepal. \\ ${ }^{2}$ Department of Radiation Oncology, Bir Hospital, Kathmandu, Nepal.
}

\begin{abstract}
Introduction: A significant proportion of patients with advanced squamous cell carcinoma of head and neck (HNSCC) are unsuitable for radical treatment and we aim to evaluate the acute toxicity, symptom relief and disease response after palliative hypofractionated radiotherapy in such patients.

Methods: A prospective observational study was conducted from November 2014 to November 2015 at the Department of Radiation Oncology, B.P. Koirala Memorial Cancer Hospital, Bharatpur in 30 patients with stage III or stage IV HNSCC who received radiotherapy of 30 Gy in 10 fractions over two weeks. Pain, dysphagia, insomnia and dyspnoea at presentation were assessed using 11 point numerical scale. Acute treatment toxicities were assessed using Common Terminology Criteria for Adverse Events (CTCAE) at the end of two weeks. After six weeks of completion of radiotherapy, percentage of symptom relief and disease response based on Response Evaluation Criteria in Solid Tumors (RECIST) were recorded. A few patients were selected for further curative radiotherapy.
\end{abstract}

Results: Common symptoms were pain (86.7\%) and dysphagia (50\%). Two-third of patients with pain and dysphagia, and about $90 \%$ patients with dyspnoea and insomnia had more than $75 \%$ symptom relief. An objective response rate of $70 \%$ and disease progression of $13.3 \%$ were observed. Acute radiation toxicities were acceptable with no grade 3 or 4 toxicities. It was observed that $46.3 \%$ of patients had mucositis, $13.3 \%$ had dysphagia, and $6.7 \%$ had hoarseness and dermatitis each.

Conclusion: Palliative radiotherapy is a suitable modality of treatment for patients with advanced HNSCC for symptom relief and tumour control, with acceptable toxicity.

Keywords: Hypofractionated Radiotherapy, Head and Neck Cancer, Palliative Radiotherapy.

\section{Introduction}

Globally, over 650,000 cases and 333,000 deaths occur due to head and neck cancer every year. ${ }^{1}$ More than $90 \%$ of head and neck cancers are squamous cell carcinoma arising from the squamous epithelium. ${ }^{2}$ Squamous cell carcinoma of head and neck (HNSCC) accounts for more than one-fourth of overall cancer burden in the developing countries. ${ }^{3}$ In general, HNSCC is treated using the single modality for early disease (stages I and II) but may require multimodality therapies for advanced stage (stages III and IV). ${ }^{4}$ The choice of the treatment modality depends on the site and stage of the disease as well as the overall health status of the patient. A significant number of patients present in the advanced incurable stage and are unfit for radical treatment with surgery or chemoradiotherapy because of advanced loco-regional disease, significant comorbidities, poor performance status, distant metastatic disease, or a combination of these factors, which carries a poor prognosis. Attempts to treat such patients with curative intent with aggressive

Correspondence

Dr. Deepa Gautam, Department of Radiation Oncology, B. P. Koirala Memorial Cancer Hospital, email: gautamdeepa09@yahoo.com 
multimodality treatment or intense radiotherapy regimens have been unsuccessful until now. ${ }^{5,6}$ There is a necessity of some form of treatment measures to alleviate their distressing symptoms and control the disease. $^{7}$ Palliative hypofractionated radiotherapy could be an effective modality to lessen the symptoms, control the disease progression, and overall improve the quality of life in those patients who are unsuitable for other anticancer measures as well as the long duration conventional radiotherapy.

Despite the lack of consensus guidelines, various treatment regimens have been practiced in the advanced HNSCC with acceptable results. ${ }^{8}$ In the developing countries with a limited number of radiotherapy centers and most of the patients traveling hundreds of kilometers to seek treatment, shortening the treatment duration reduces the economic burden to the patients as well as a burden to health resources. In the current study, we aim to evaluate the role of palliative hypofractionated regimen in advanced HNSCC.

\section{Methods}

A hospital-based prospective observational study was conducted from November 2014 to November 2015 in patients of head and neck cancer attending B.P. Koirala Memorial Cancer Hospital, Bharatpur after taking ethical clearance from Institutional Review Board (IRB), National Academy of Medical Sciences (NAMS), Kathmandu, Nepal. All patients of head and neck cancer attending the joint clinic of Radiation Oncology, and Otorhinolaryngology and head neck surgery were subjected to the proper pre-treatment evaluation and the study cases were selected as per the inclusion criteria.

Detailed histories, complete physical examination including Eastern Co-operative Oncology Group (ECOG) performance status ${ }^{9}$ as well as endoscopic and radiological findings were recorded in a predesigned proforma and Tumor Node Metastasis (TNM) classification of the tumor was done. Biopsy-proven cases of squamous cell carcinoma of the oral cavity, oropharynx, hypopharynx, and larynx American Joint Committee on Cancer (AJCC) Stage III disease10 with $\mathrm{ECOG}^{9}$ performance status 3 and AJCC Stage IV disease ${ }^{10}$ with ECOG performance status 2 and 3, surgically unresectable, medically inoperable were included. Tumors of salivary glands, paranasal sinus, nasopharynx, recurrent disease, and those with a history of prior radiotherapy in head and neck region or chemotherapy were excluded. The distressing symptoms at the time of presentation namely, pain, dysphagia, insomnia, and dyspnoea, were assessed using an 11 point numerical scales and graded as mild (score 1-3), moderate (4-6) and severe (>6). ${ }^{11}$

Radiotherapy was delivered by 6 Mega Voltage (MV) Linear Accelerator. Patients were immobilized in a supine position in a head-and-neck thermoplastic mask and conventionally simulated. Treatment included two lateral opposed parallel fields, with a matched anterior low neck field, as needed for the supraclavicular region, with the radiation portals being individualized depending upon the extent and location of the primary tumor and the involved lymph nodes. This included the gross disease with a $1.5 \mathrm{~cm}$ margin without prophylactic nodal irradiation. The total dose of radiotherapy up to 30Gy in 10 fractions, five fractions per week, over two weeks was given.

Supportive measures such as enteral feeding for nutrition, analgesics as per the $\mathrm{WHO}$ pain ladder ${ }^{12}$, cough sedatives, anxiolytics were provided appropriately. Toxicities were carefully assessed, managed, and recorded as perCommon Terminology Criteria for Adverse Events (CTCAE) ${ }^{13}$ at the end of two weeks. After six weeks of completion of radiotherapy, detailed assessment of symptom relief as a percentage of symptom relief compared to baseline and evaluation of tumor response as per Response Evaluation Criteria in Solid Tumors (RECIST) ${ }^{14}$ based on clinical, endoscopic and radiological findings were done. The patients achieving more than $50 \%$ relief of symptoms and partial response at the primary and nodal sites with improvement in the performance status were referred for further radiotherapy of curative intent to achieve a total a equivalent dose of 66 to $70 \mathrm{~Gy}$.

\section{Results}

A total of 30 patients of HNSCC were registered for the study. (Table1) The age of the patients ranged from 38 years to 87 years with a median age of 65 years. The four specific sites were included with an oral cavity 


\section{ВРКМСH}

accounting for the maximum cases and oropharynx for the least. Around half of the patients were of stage IVA. (Table1) More than two-thirds of patients had ECOG performance status ${ }^{2}$. Grading of distressing symptoms at the time of presentation is shown in Table 2.

Table 1: Baseline variables of the patients

\begin{tabular}{|l|l|l|}
\hline \multicolumn{1}{|c|}{ Variables } & \multicolumn{1}{c|}{$\begin{array}{c}\text { Number of } \\
\text { patients (\%) }\end{array}$} \\
\hline \multirow{2}{*}{ Gender } & Male & $23(76.7)$ \\
\cline { 2 - 3 } & Female & $7(23.3)$ \\
\hline ECOG performance & 2 & $21(70)$ \\
\cline { 2 - 3 } status & 3 & $9(30)$ \\
\hline \multirow{4}{*}{ Tumor site } & Oral cavity & $11(36.7)$ \\
\cline { 2 - 3 } & Oropharynx & $4(13.3)$ \\
\cline { 2 - 3 } & Hypopharynx & $5(16.7)$ \\
\cline { 2 - 3 } & Larynx & $10(33.3)$ \\
\hline \multirow{5}{*}{ tumor stage } & III & $2(6.7)$ \\
\cline { 2 - 3 } & IVA & $16(53.3)$ \\
\cline { 2 - 3 } & IVB & $9(30)$ \\
\cline { 2 - 3 } & IVC & $3(10)$ \\
\hline
\end{tabular}

Table 2: Symptom grading by 11 point numerical scale

\begin{tabular}{|l|l|l|l|}
\hline Symptoms & Mild n(\%) & $\begin{array}{c}\text { Moderate } \\
\mathbf{n}(\%)\end{array}$ & $\begin{array}{c}\text { Severe } \\
\mathbf{n}(\%)\end{array}$ \\
\hline Pain & $2(7.7)$ & $17(65.4)$ & $7(26.9)$ \\
\hline Dysphagia & $2(13.3)$ & $11(73.3)$ & $2(13.4)$ \\
\hline Insomnia & $1(7.1)$ & $11(78.6)$ & $2(14.3)$ \\
\hline Dysphonia & $1(10)$ & $5(50)$ & $4(40)$ \\
\hline
\end{tabular}

Treatment-related toxicities are demonstrated in Table 3. All of the patients had either grade 1 or grade 2 toxicities. (Table 3)

Table 3: Acute treatment toxicities at the end of two weeks

\begin{tabular}{|l|l|l|l|l|}
\hline \multicolumn{1}{|c|}{$\begin{array}{c}\text { Acute } \\
\text { Toxicities }\end{array}$} & \multicolumn{1}{|c|}{$\begin{array}{c}\text { Total } \\
\mathbf{n}(\%)\end{array}$} & \multicolumn{1}{|c|}{ Grade 1 } & \multicolumn{1}{|c|}{$\mathbf{2}$} & \multicolumn{1}{|c|}{$\begin{array}{c}\text { Grade } \\
\mathbf{3 , 4}\end{array}$} \\
\hline Mucositis & $14(46.3)$ & $12(85.8)$ & $2(14.2)$ & 0 \\
\hline Dysphagia & $4(13.3)$ & $1(25)$ & $3(75)$ & 0 \\
\hline Hoarseness & $2(6.7)$ & $2(100)$ & 0 & 0 \\
\hline Dermatitis & $2(6.7)$ & $2(100)$ & 0 & 0 \\
\hline
\end{tabular}

Distressing symptoms at presentation and their relief are depicted in Table 4. A significant number of patients had more than $75 \%$ relief of their symptoms.
NEPALESE JOURNAL OF CANCER (NJC)

Table 4: Symptom relief at the end of six weeks of completion of radiotherapy

\begin{tabular}{|l|l|l|l|l|}
\hline Symptoms & \multicolumn{1}{|c|}{$\begin{array}{c}\text { Total } \\
\mathbf{n}(\%)\end{array}$} & $\begin{array}{c}<\mathbf{5 0 \%} \\
\text { relief }\end{array}$ & $\begin{array}{c}\mathbf{5 0 - 7 5 \%} \\
\text { relief }\end{array}$ & \multicolumn{1}{|c|}{$\begin{array}{c}\mathbf{7 5 \%} \\
\text { relief }\end{array}$} \\
\hline Pain & $26(86.7)$ & $6(23.1)$ & $3(11.5)$ & $17(65.4)$ \\
\hline Dysphagia & $15(50)$ & $3(20)$ & $2(13.4)$ & $10(66.6)$ \\
\hline Insomnia & $14(43.3)$ & 0 & $1(7.1)$ & $13(92.9)$ \\
\hline Dyspnoea & $10(33.3)$ & $1(10)$ & 0 & $9(90)$ \\
\hline
\end{tabular}

On evaluation after six weeks of treatment, as per RECIST 19(63.3\%) patients had partial response, 2 (6.7\%) had complete response, 5 (16.7\%) had stable disease, and the remaining four(13.3\%) had disease progression. After evaluating the symptom relief and tumor response in the patients after six weeks, 11 (36.7\%) patients who achieved more than $50 \%$ relief of symptoms and partial response at the primary and nodal sites with the improvement in the performance status were referred for further dose escalation to receive the radiobiologically equivalent dose of 66 to 70 Gy.

\section{Discussion}

Advanced head and neck cancers are a major cause of cancer-related deaths in developing countries.1 The treatment of those groups of patients has led to a great challenge to the treatment providers because of the troublesome symptoms, disease extent, and poor performance status. With the aim of symptom alleviation and disease control, researchers have used different radiotherapy regimens with a wide range of variation in total dose, dose per fraction and treatment duration ranging from three days to seven weeks. ${ }^{8}$

Weissberg et al. ${ }^{15}$ assessed conventionally fractionated versus hypofractionated palliative external beam radiation therapy schedules in patients with locally recurrent or advanced head and neck cancers comparing 60 to $70 \mathrm{~Gy}$ in six to seven weeks against 40 to $48 \mathrm{~Gy}$ in 64 patients. No variances were observed in tumor control, acute side effects, or long term sequelae and since then various short-course hypofractionated regimens have emerged in advanced HNSCC. This study presents the role of hypofractionated radiotherapy of 30 Gy in 10 fractions over two weeks in advanced squamous cell carcinoma of head and neck. The similar treatment regimens had been evaluated earlier by Ali et al. ${ }^{16}$ and Ghosal et al. ${ }^{17}$ 
The site-wise variation of disease in the study population differed in various studies. The commonest site was oropharynx in the studies done by Porceddu et al. ${ }^{18}$ and Al-Mamdani et al. ${ }^{19}$ while Ali et al. ${ }^{16}$ observed that the larynx was the commonest site that accounted for $37 \%$ of cases. In our study, we observed that the oral cavity was the commonest site of the disease accounting for $36.7 \%$ of cases as similar to the study published by Corry et al. ${ }^{20}$ The reference studies showed the variability in the stage-wise distribution of the cases. Maximum number of cases were from the stage IVA in a majority of the studies. ${ }^{18-21}$ In our study, about half (53.3\%) were of stage IVA disease which accounted for the maximum number.

Since performance status is one of the important criteria for choosing the modality of treatment in the patient, previous studies have defined the performance status of the patients in inclusion criteria using either the Karnofsky scale or ECOG. We included the patients with ECOG performance status of 2 and 3 in our study. For stage III disease, we strictly included the patients with a performance status of 3 to receive the hypofractionated radiotherapy.

Among the distressing symptoms evaluated in the current study, the pain was the commonest $(86.7 \%)$ followed by dysphagia occurring in half of the patients, insomnia in $43.3 \%$ of patients, and dyspnoea in $33.3 \%$ of patients. Among the patients with pain, two-third had moderate and one fourth had a severe grade. Likewise, among the cases with dysphagia and insomnia, nearly three-fourth patients suffered from moderate grade. Half of the patients with dyspnoea had moderate grade and $40 \%$ had a severe grade.

The evaluation of symptom relief in the reference studies was done after a month of completion of radiotherapy, however, we evaluated the symptom relief after 6 weeks for the convenience of the patients so that the evaluation of tumor response could also be done at the single sitting and the repeated visits could be avoided. We observed that all the patients had symptomatic relief to various extents. About two-thirds of patients with pain and dysphagia, $92.9 \%$ of patients with insomnia, and $90 \%$ of patients with dyspnoea experienced more than $75 \%$ relief of their symptoms. In studies by Ali et al. ${ }^{16}$ and Ghosal et al. ${ }^{17}$, more than $50 \%$ relief of symptoms was observed in all patients with pain and about $90 \%$ of patients with dysphagia and insomnia. All patients with dyspnoea had more than $75 \%$ of the relief of their symptoms. Paliwal et al. $^{22}$ observed appreciable relief of pain in nearly two-thirds of patients and around three-fourth patients with dysphagia, and dyspnoea. Similarly, in the study by Mohanti et al. ${ }^{23}$, they reported good symptomatic relief of more than $50 \%$ in over half of the patients, with pain, dysphagia, hoarseness, and cough as well as $47 \%$ patients with otalgia and $76 \%$ with respiratory distress. A multicenter Australian study on 35 patients treated with a hypofractionated radiotherapy regimen observed that the quality of life improvement and symptom control was reported in about two-thirds of the patients. ${ }^{18}$

Acute radiation toxicities were acceptable with none of the patients experiencing grade 3 or 4 toxicities. It was observed that nearly half of the patients had mucositis, among which $85.8 \%$ had grade 1 mucositis. Similarly, $13.3 \%$ of patients had dysphagia among which threefourth of patients had grade 2 dysphagia. Hoarseness and dermatitis were observed in $6.7 \%$ of the patients each and all were of grade 1 . The study by Ali et al. ${ }^{16}$ and Ghosal et al. ${ }^{17}$ observed that grade 1 mucositis was seen in about two-thirds of patients and grade 2 mucositis in remaining patients. The study by Cory et al..$^{20}$ with the QUAD-SHOT regimen also demonstrated that the toxicities observed in their patients were acceptable with most them showing grade 1 and 2 toxicities and few grade 3 toxicities. In contrast to the above studies, the study by Porceddu et al. ${ }^{18}$ demonstrated the occurrence of grade 4 toxicity in $6 \%$ of patients in the form of dysphagia.

Ali et al. ${ }^{16}$, observed the overall objective response rate of $73 \%$ with $26 \%$ of complete response and $47 \%$ of partial response in their research. A similar result of overall objective response rate of $73 \%$ was observed following the Christie scheme ${ }^{19}$ but the complete and partial response rates were $45 \%$ and $44 \%$ respectively. Our study showed the result almost similar to these studies with $70 \%$ of the overall objective response rate. There was $6.7 \%$ of complete and $63.3 \%$ of partial response rates, $13.3 \%$ had disease progression remaining $16.7 \%$ had stable disease. The various studies have shown that there was a great relief of the distressing symptoms, as well as improvement 
in the performance status of the patients following the completion of short-course hypofractionated radiotherapy. Those achieving the partial response at the tumor and nodal sites were further escalated to the conventional fractionation regime biologically equivalent total dose of $66 \mathrm{~Gy}$ to $70 \mathrm{~Gy}$. In the study by Ali et al. ${ }^{16}, 27 \%$ of patients were further escalated to the total radiobiologically equivalent dose of 66 Gy. Similarly, in the study by Mohanti et al. ${ }^{23}$, after completion of hypofractionated radiotherapy, $30 \%$ of patients received further conventional radiotherapy. Likewise, in our study, after evaluating the symptom relief and tumor response in the patients at six weeks, $36.7 \%$ of patients were referred for further dose escalation to receive the radiobiologically equivalent dose of 66 to $70 \mathrm{~Gy}$.

Newer radiotherapy techniques such as 3D-CRT, IMRT, SBRT have provided promising results compared to conventional modality including our study. However, hypofractionated radiotherapy through conventional technique can also be considered for relief of distressing symptoms as well as to achieve disease control, with acceptable toxicity.

\section{Conclusion}

Hence, it is concluded from this study that the hypofractionated regimen of 30 Gy in 10 fractions over 2 weeks is a suitable option for patients with advanced unresectable or medically inoperable squamous cell carcinoma of head and neck or for patients with poor performance status, for symptom relief and tumor control. Compliance towards the treatment is excellent and results in high objective response rates, greater improvement in distressing symptoms, with acceptable toxicity. It also helps in selecting the patients for further dose escalation.

\section{Limitations of the Study}

Due to time constraints, a limited number of cases were enrolled for the study, tumor control and toxicities could not be assessed beyond six weeks and also the overall survival could not be assessed.

\section{References}

1. Bray F, Ferlay J, Soerjomataram I, Siegel RL, Torre LA, Jemal A. Global cancer statistics 2018: GLOBOCAN estimates of incidence and mortality worldwide for
36 cancers in 185 countries. CA Cancer J Clin 2018; 68:394.

2. Vigneswaran N, Williams MD. Epidemiologic trends in head and neck cancer and aids in diagnosis. Oral Maxillofac Surg Clin North Am. 2014;26(2):123-141.

3. Joshi P, Dutta S, Chaturvedi P, Nair S.Head and Neck Cancers in Developing Countries: Rambam Maimonides Med J. 2014;5(2)

4. Ridge JA, Glisson BS, Lango MN, Feigenberg S. Head and neck tumours. In : Pazdur R, Wagman LD, Camphausen KA, Hoskins WJ, editors. Cancer management: A multidisciplinary approach. 12th ed. New York: UBM Medica LLC; 2009:1-6

5. Carvalho AL, Salvajoli JV, Kowalski LP. A comparison of radiotherapy or radiochemotherapy with symptomatic treatment alone in patients with advanced head and neck carcinomas. Eur Arch Otorhinolaryngol 2000;257:164-7

6. Dobrowsky W, Naude J. Continuous hyperfractionated accelerated radiotherapy with/ without mitomycin $\mathrm{C}$ in head and neck cancers. Radiother Oncol 2000;57:119-24.

7. Agarwal JP, Nemade B, Murthy V, Ghosh-Laskar SG, Budrukkar A, Gupta T et al. Hypofractionated, palliative radiotherapy for advanced head and neck cancer: Radiotherapy and Oncology 2008;89:51-56

8. Grewal AS, Jones J, Lin A. Palliative Radiation Therapy for Head and Neck Cancers. Int J Radiat Oncol Biol Phys. 2019;105(2):254-266.

9. Zubrod CG, Scheiderman M, Frei E, Brindley C, Gold LG, Shnider B, et al. Cancer - appraisal of methods for the study of chemotherapy of cancer in man: thiophosphamide. J. Chronic. Dis.1960; 11:733.

10. American Joint Committee on Cancer. AJCC cancer staging manual. 7th ed. Springer, New York; 2010

11. Price DD, Harkins SW. Psychosocial approaches to pain measurement and assessment. In: Turk D, Melzac R, editors. Handbook of Pain Assessment. New York: Guildford Press; 1992;165-79

12. Cancer pain relief and palliative care, Report of a WHO expert committee. WHO Tech Rep Ser 804, 1990; $1-73$

13. National Cancer institute. National Cancer Institute Common Terminology Criteria for Adverse Events, NCI-CTCAE v.4 (2010).Available: http://evs.nci.nih. 
gov/ftp1/CTCAE/About.html. Accessed: December 27, 2014

14. Eisenhauer EA1, Therasse P, Bogaerts J, Schwartz LH, Sargent D, Ford R et al. New response evaluation criteria in solid tumours: revised RECIST guideline (version 1.1). Eur J Cancer. 2009;45(2):228-47

15. Weissberg JB, Son YH, Percarpio B, Fischer JJ. Randomised trial of conventional versus high fractional dose radiation therapy in the treatment of advanced head and neck cancer. Int J Radiat Oncol Biol Phys 1982;8:179-85

16. Ali MY, Alam MSS, Mannan MA, Asaduzzaman AKM, Khan MA, Islam SMJ: Short course palliative radiotherapy in locally advanced squamous cell carcinoma of head and neck. JAFMC Bangladesh. 2010; 6:1

17. Ghoshal S, Patel F, Mudgil N, Bansal M, Sharma S: Palliative radiotherapy in locally advanced head and neck cancer-a prospective trial: Indian J Palliat Care 2004; 10(1): 19-23

18. Porceddu SV, Rosser B, Burmeister BH, Jones M, Hickey B, Baumann $\mathrm{K}$ et al. Hypofractionated radiotherapy for the palliation of advanced head and neck cancer in patients unsuitable for curative treatment-hypotrial. Radiother Oncol 2007;85(3):456-62
19. Al-Mamgani A, Tans L, Van rooij $\mathrm{PH}$, Noever I, Baatenburg de jong RJ, Levendag PC. Hypofractionated radiotherapy denoted as the "Christie scheme": An effective means of palliating patients with head and neck cancers not suitable for curative treatment. Acta Oncol 2009;48(4):562-70

20. Corry J, Peters LJ, Costa ID, Milner AD, Fawns H, Rischin D et al. The'QUAD SHOT': A phase II study of palliative radiotherapy for incurable head and neck cancer. Radiother Oncol 2005;77(2):137-42.

21. Kancherla KN, Oksuz DC, Prestwich RJD, Fosker C, Dyker KE, Coyle CC et al. The Role of Split-course Hypofractionated Palliative Radiotherapy in Head and Neck Cancer. Clin Oncol 2011;23:141-48

22. Paliwal R, Patidar AK, Walke R, Hirapara P, Jain S, Bardia MR. Palliative Hypofractionated Radiotherapy in Locally Advanced Head and Neck Cancer With Fixed Neck Nodes. Iran J Cancer Prev.2012; 5(4): 178-82.

23. Mohanti BK, Umapathy H, Bahadur S, Thakar A, Pathy S. Short-course palliative radiotherapy of 20 Gy in 5 fractions for advanced and incurable head and neck cancer: AIIMS study. Radiother Oncol. 2004; 71(3):275-80. 